\title{
A Theranostic Imaging prototype based on SiPM detectors for nanoparticles imaging during hyperthermia
}

\author{
George Loudos ${ }^{1, a}$, Maria Georgiou ${ }^{1}$, Eleftherios Fysikopoulos ${ }^{1}$ and Eirini Fragogeorgi ${ }^{1}$ \\ ${ }^{1}$ Department of Biomedical Engineering, Technological Educational Institute of Athens, Athens, Greece
}

\begin{abstract}
The combination of imaging and therapy has opened the very promising Theranostics domain with magnetic hyperthermia being a very promising domain. However, imaging systems should not interact with the magnetic field. In this work we have tested the recent C-series of SensL SiPM with $3 \mathrm{~mm}$ pixel size, $4 \times 4$ arrays, coupled to different scintillators and irradiated with various gamma energies. The evaluation of the SiPM arrays shows that $1 \times 1 \mathrm{~mm}$ pixel size can be clearly resolved at PET energies for GAGG:Ce and CsI:Na and 1.5x1.5mm in SPECT imaging for CsI:Na. The best energy resolution was measured equal to $10.5 \%$ under $511 \mathrm{keV}$ irradiation for the 2x2mm GAGG:Ce; $16 \%$ under $511 \mathrm{keV}$ irradiation for the 1x1mm GAGG:Ce and $22 \%$ under $120 \mathrm{keV}$ irradiation for the $1 \times 1 \mathrm{~mm}$ CsI:Na. In addition, measurements with position sensitive photomultipliers have been carried out, to evaluate the effect of the magnetic field on the imaging performance of the system. While the effect of the magnetic field outside the coil is small, optimal images will be obtained if the imaging system is placed inside the coil, something that can be achieved only by using SiPMs.
\end{abstract}

\section{Introduction}

Radiolabelled magnetic nanoparticles are challenging agents for drug delivery in medicine. They can penetrate biological barriers, carry drugs on the target site, while minimizing dose in other organs. Their magnetic properties allow them to be heated and either allow controlled drug release and/or transfer heat to cancer cells, thus induce a hyperthermic effect. When nanoparticles are radiolabelled it is possible to image them, using standard molecular imaging SPECT or PET techniques [1]. The combination of imaging and therapy has opened the very promising Theranostics domain. Simultaneous nuclear imaging during hyperthermia can provide insights in the biological process that occur when nanoparticles are heated [2]. In this way it is possible to monitor the successful organ/tumor targeting, drug release and/or real time response to therapy. This approach is by far superior when compared to the use of conventional anatomic or functional modalities, which can monitor the long term therapeutic effect.

A typical imaging system consists of a coil, which induces a magnetic field. However, conventional photon detectors such as photomultipliers cannot operate in magnetic fields. Thus, a theranostic imaging system should be designed in a way that such interactions are minimized and do not introduce artifacts in the obtained images. Silicon Photomlutipliers (SiPM) are magnetic insensitive detectors, which have been widely used in the PET/MR domain [3]. Their compact characteristics, as well as their compatibility with magnetic fields make them promising candidates for the design and development of small imaging systems, which are compatible with hyperthermia equipment. According to our knowledge, this is the first time that SiPMs are tested for such an application.

Position Sensitive Photomultipliers Tubes (PSPMTs) are considered as the standard detectors of choice for the development of small animal imaging systems [4]. However, their compatibility with the magnetic field outside the coil of the hyperthermia system has not been tested yet. In addition, since they are not very compact they cannot be used for the design of optimal detectors in terms of geometry and subsequently performance. For this reason we have tested the performance of a PSPMT close to the magnetic field of a hyperthermia system. We have also developed different detector modules based on SiPMs, which can serve as the basis for the development of SPECT and PET systems that can be used inside the magnetic field of the hyperthermia coil.

\section{Methods and materials}

The hyperthermia setup is based on a MP $6 \mathrm{~kW}$ instrument (Fives Celes, Lautenbach, France); The magnetic field is set by adjusting the voltage in the power supply, the frequency with an appropriate copper coil $(\varnothing$ $110 \mathrm{~mm}$, three turns), and an inductor set-up $[\mathrm{H} 0=25$ $(\mathrm{kA} / \mathrm{m}), \mathrm{f}=173(\mathrm{kHz})]$. The system has been used for the study of nanoparticles heating, as well as in vivo hyperthermia in small mice. To monitor the temperature increase in mice and nanoparticles we use a

\footnotetext{
${ }^{\text {a }}$ Corresponding author: author@e-mail.org
} 
ThermaCAM ${ }^{\mathrm{TM}}$ E300 (FLIR) (FLIR Systems Inc., Meer, Belgium) infrared camera (FLIR Systems, Inc). In all cases the animal studies are performed in compliance with European legislation and were approved by the relevant Greek authorities.

In Figure 1 a typical image of the temperature increase in a mouse injected with nanoparticles on the tumor region is shown. In this example, the mouse is placed inside the coil and direct nanoparticles injection on the tumor is performed; the local heating is evident from the obtained images, since the tumor are is visualized as a hot spot.

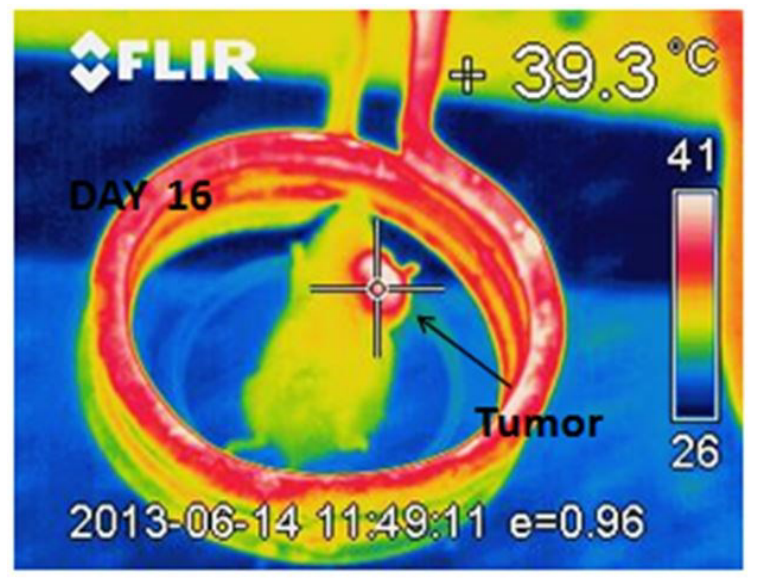

Figure 1. Temperature increase in a tumor bearing mouse injected with magnetic NPs, monitored with a thermocamera.

There are many ways to design a SPECT or PET imaging system in combination with a magnetic hyperthermia system as it can be seen in Figure 2. In this figure the detector heads of a PET system are visualized as simple blocks, although more compact shapes are possible. The one or more detector heads can be placed either inside or outside the coil. They can also image the field of view from $0 \mathrm{o}$ or $90 \mathrm{o}$, depending on the animal placement, as well as possible interactions with the hyperthermia system.
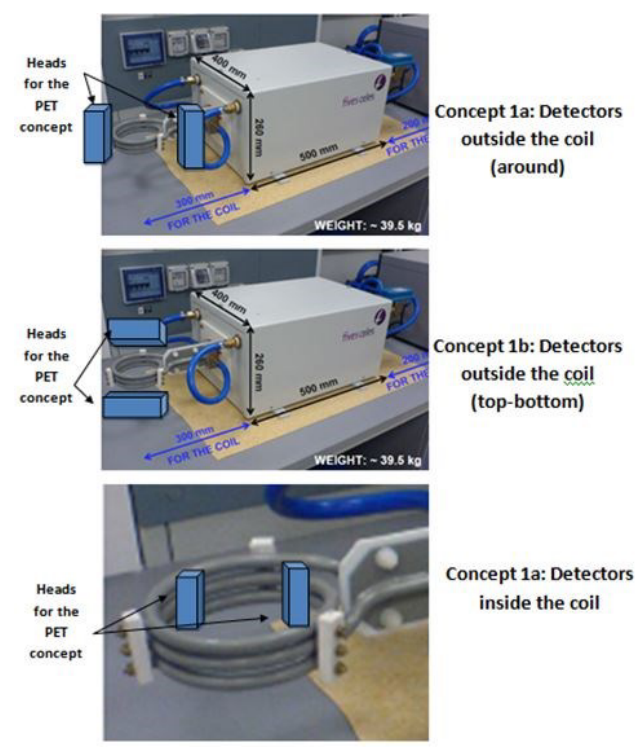

Figure 2. Alternative concepts of combining the hypertermic device and the proposed imaging modules.
Theoretically, the magnetic field outside the coil is not very strong and is likely that will not interfere with the detectors. However, to obtain high resolution images, the distance between the detector and the object to be imaged should be minimized. This is possible with the use of detectors, which are not affected by magnetic field, such as SiPMs. For exploring both nuclear medicine modalities, we tested both a SPECT and a PET detector module.

\subsection{SiPMs for SPECT components}

The SPECT component is based on the C-series of SensL SiPM with $3 \mathrm{~mm}$ pixel size, $4 \mathrm{x} 4$ arrays. The 16 output signals of the SiPM array are further reduced to 4 position signals, then amplified and then digitized using free running ADCs. The sampling rate was $40 \mathrm{MHz}$. A Field Programmable Gate Array (FPGA) Spartan 6 LX150T is used for triggering and processing of the four digitized position signals. Data are transferred to a standard PC via Ethernet link.

The evaluation of the SiPM arrays was performed using a pixilated CsI:Na crystal with dimensions $1.0 \times 1.0 \times 5.0 \mathrm{~mm}^{3}$ and a Na22 source. Three different readout strategies were used, namely (i) a symmetric resistive voltage division circuit (Voltage-SCD), secondly (ii) a symmetric resistive charge division circuit (Charge-SCD) and thirdly (iii) a charge division multiplexing resistor network (DPC) [5]

\subsection{SiPMs for PET components}

The PET component is based again on the C-series of SensL SiPM with $3 \mathrm{~mm}$ pixel size, $4 \mathrm{x} 4$ arrays. The 16 output signals of the SiPM array are further reduced to 4 position signals through a two-stage charge division resistive network and then amplified and then digitized using free running ADCs. The sampling rate was 40 MHz. A Field Programmable Gate Array (FPGA) Spartan $6 \mathrm{LX} 150 \mathrm{~T}$ is used for triggering and processing of the four digitized position signals. Data are transferred to a standard PC via Ethernet link. The evaluation of the SiPM arrays was performed using a $3 \times 3 \times 5 \mathrm{~mm}^{3}$ GAGG:Ce scintillator and a $\mathrm{Na}^{22}$ source.

\subsection{Measurements with PSPMTs}

To evaluate the interaction of the PSPMTs with the magnetic field we used a simple set up, based on the R8900U-00-C12 PSPMT (Hamamatsu, Japan), coupled to a $\operatorname{CsI}(\mathrm{Na})$ pixelated scintillator with $1 \times 1 \times 5 \mathrm{~mm}^{3}$ pixel size, $0.2 \mathrm{~mm}$ septa and $1 \mathrm{~mm}$ glass entrance window (Hilger, U.K.). The 12 outputs of the PSPMT were reduced to 4 position signals using a resistive network and were connected to a four channel data acquisition system based on compact ADCs and the Spartan 3E FPGA. This set up was very compact and it was placed inside a copper box in order to be electromagnetically shielded. A small low activity $\mathrm{Tc}^{99 \mathrm{~m}}$ source was placed in the center of the field of view of the detector at a fixed 
position of $10 \mathrm{~cm}$ to irradiate uniformly the entire field of view.

The entire set up, the source and the copper box, was placed at variable distances of $50 \mathrm{~cm}$ to $10 \mathrm{~cm}$ from the center of the field of the hyperthermia system, both on $\mathrm{x}$ and $\mathrm{z}$ axis. The frequency that was used was $400 \mathrm{kHz}$ and raw images of the PSPMT were recorded for $15 \mathrm{~min}$ with the hyperthermia system on. The standard setup where the measurements took place, are shown in Figure 3.

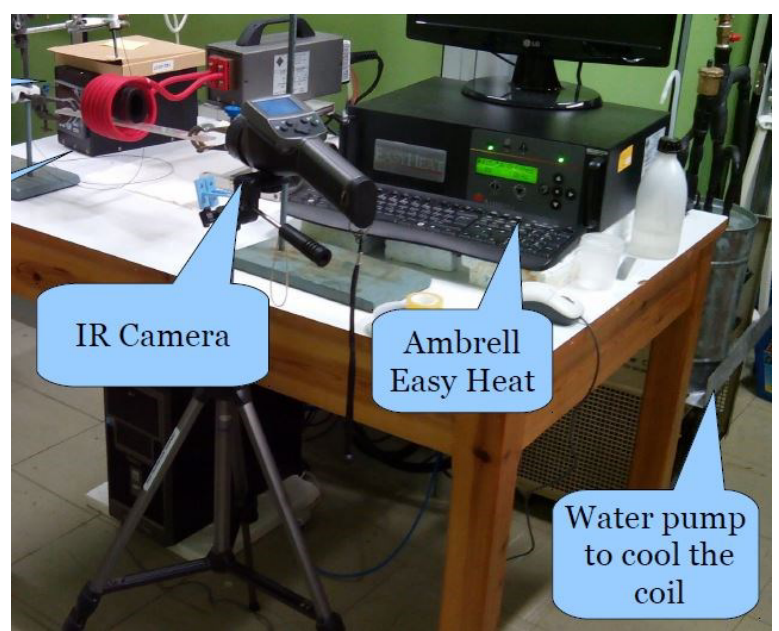

Figure 3. Setup of hyperthermia system.

\section{Results}

\subsection{SiPMs for SPECT components}

The evaluation of the SiPM arrays shows that crystals of CsI:Na with dimensions $1.0 \times 1.0 \mathrm{~mm}$ can be clearly resolved. The best energy resolution was measured equal $22 \%$ under $120 \mathrm{keV}$ irradiation for the $1 \times 1 \mathrm{~mm}^{2} \mathrm{CsI}: \mathrm{Na}$. The obtained raw images and typical line profiles for the three tested circuits are shown in Figure 4.
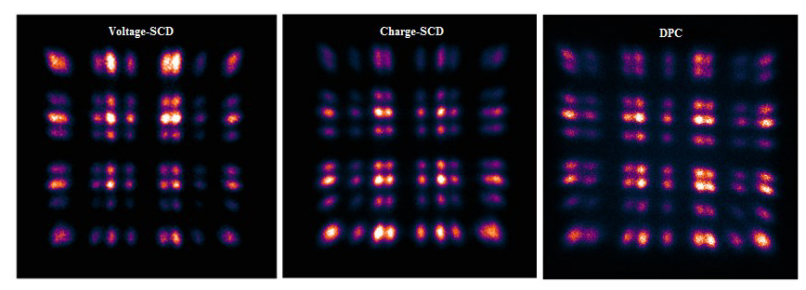

Crystal CsII(Na) $1 \times 1 \times 5 \mathrm{~mm}^{3}$

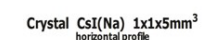

Crystal CsI(Na) $1 \times 1 \times 5 \mathrm{~mm}^{3}$
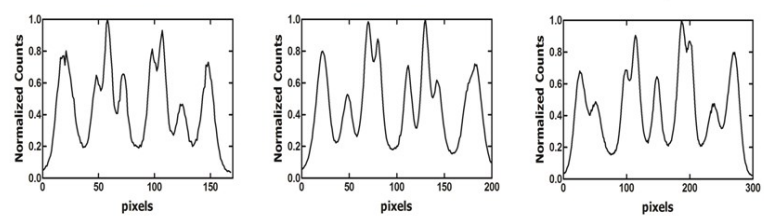

Figure 4. Raw images (up) and horizontal profiles (bottom) of the $1 \times 1 \times 5 \mathrm{~mm}^{3}$ CsI:Na scintillator, obtained by the VoltageSCD (left), the Charge-SCD (center) and the DPC (right) readout circuits.

Since the $1 \times 1 \mathrm{~mm}^{2}$ pixels can be clearly identified, it is possible to apply a correction look up table and follow all standard calibration steps, in order to formulate corrected images. Thus this module can be used as a SPECT component, which is compatible with the magnetic field of the hyperthermia system.

\subsection{SiPMs for PET components}

The evaluation of the PET component with the GAGG:Ce scintillator has shown that all crystal pixels are very clearly identified with a mean peak to valley ratio equal to 40 . The measured energy resolution under $511 \mathrm{keV}$ irradiation was equal to $10.5 \%$.
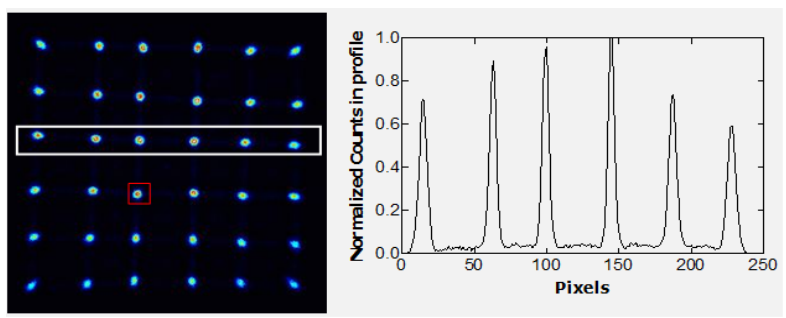

Figure 5. Raw image of the $6 \times 6$ GAGG:Ce scintillator array and a horizontal profile of the central scintillator elements produced under $511 \mathrm{keV}$ excitation.

These results, show that it is possible to use the combination of SiPMs and GaGG:Ce, as the basic component of a PET system, which can operate in combination with the magnetic field of the hyperthermia system.

\subsection{Measurements with PSPMTs}

The experimental measurements with the shielded PSPMT module showed that it is possible to use this component outside the coil, with almost negligible interference. In Figure 6 the raw images, typical line profiles and the energy spectra of a typical crystal pixel are shown, when the detector is placed at $10 \mathrm{~cm}$ (upper row) and $50 \mathrm{~cm}$ (bottom row). As it is observed all crystal pixels are clearly resolved in both cases. A deterioration in peal to valley ratio (from 11:1 to 7.4:1) and in energy resolution (from $15.4 \%$ to $16 \%$ ) is observed, which however is not expected to affect the image quality.

\section{Discussion}

The presented results show that the SiPM can perform as efficient detector modules both in SPECT and PET, in order to allow the design of flexible geometries, which can be combined with the coil of a magnetic field of a hyperthermia device. This is the first time that SiPMs are proposed for such an application, since until now their use was in the field of PET/MR and SPECT/MR research. In this work basic SiPM modules were used, along with standard crystals. However, in order to design and develop a more advanced Theragnostic system, it is possible to take advantage of even smaller and more compact SiPMs coupled to smaller crystal pixels. The experimental evaluation of such a system will require placement of such modules inside the magnetic coil. 

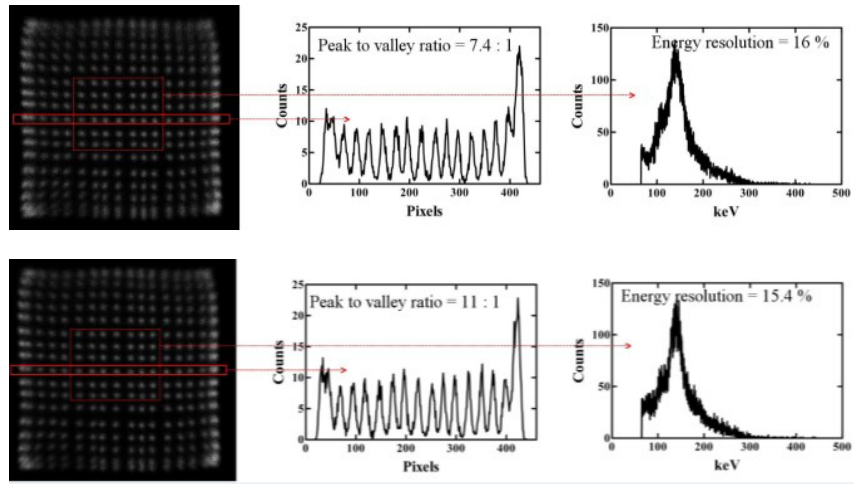

Figure 6. Raw images, typical line profiles and the energy spectra of a typical crystal pixel, when the detector is placed at $10 \mathrm{~cm}$ (upper row) and $50 \mathrm{~cm}$ (bottom row), irradiated with a Tc99m source.

The experiment with PSPMTs has shown that it is possible to use conventional detectors outside the coil and still obtain acceptable images. For this reason we aim to continue the experimental evaluation both for SPECT and PET. For SPECT, it is possible to use a pinhole collimator, so that the camera is still placed at a large distance from the coil and derive images by magnifying the object to be imaged. For PET, two or more heads can be placed around the coil and provide optimal resolution at the center of the field of view, which is located inside the coil.

Taking into account the large number of variable parameters, such as supply current, capacitors of the hyperthermia system, the size and the geometry of the coil, there are several parameters that need to be experimentally studied. For this reason it is important to develop a proper simulation model, which can be used to study in silico the contribution of all the components and variables, which can affect the design of the proposed theranostic system. Currently, such a model is developed using Comsol [6] for simulating the coil and GATE [7] for simulating the optimal design of the imaging components, when placed inside and/or outside the coil.

\section{Acknowledgment}

This research has been co-funded by the European Union (European Social Fund) and Greek national resources under the framework of the "ARISTEIA II" project HYPERGNOSTIC code 4309 of the "Education \& Lifelong Learning” Operational Programme.

\section{References}

1. George Loudos et al, Eur J Radiol. 78(2), 287-295 (2011).

2. B. Thiesen and A. Jordan, Int J Hyperthermia. 24, 467-474 (2008).

3. E. Roncali and S.R. Cherry, Ann. Biomed. Eng. 39, 1358-1377 (2011).

4. Pani et al, Nucl. Instr. Meth. Phys. Res. B 213, 197205 (2004).
5. S. David, et al, Nucl. Instr. Meth. Phys. Res. A 702, 121-125 (2012).

6. https://www.comsol.com/

7. Sarut et al, Med. Phys. 41, (2014). 